\title{
A Study of Various PAPR Reduction Techniques for Optical OFDM Systems
}

\author{
Dhanvi Gupta \\ PEC University of Technology \\ Chandigarh, India
}

\author{
Divya Dhawan \\ PEC University of Technology \\ Chandigarh, India
}

\begin{abstract}
In present times, OFDM has emerged as a key area of research in optical communication. Ofdm is a modulation technique by which high data rates can be achieved by sending data simultaneously over multiple subcarrier frequencies. However the main problem of OFDM is high PAPR value. High PAPR enhances non linear effects in optical fibers as the effective refractive index of optical fiber depends upon the value of input power. Various techniques have been deployed to reduce PAPR .All techniques have their inherent advantages and limitations. This paper presents a review on some of the important PAPR reduction techniques for optical OFDM systems.
\end{abstract}

\section{Keywords}

Orthogonal Frequency Division Multiplexing (OFDM), Partial Transmit Sequence (PTS), Peak to Average Power Ratio (PAPR), Selected Mapping (SLM), Sliding Norm Transform (SNT).

\section{INTRODUCTION}

OFDM is emerging as promising research area for high speed optical communication systems. OFDM is a frequency division multiplexing based multiple subcarrier modulation technique by which high data rates can be obtained by sending data simultaneously at lower data rates over multiple orthogonal subcarriers. OFDM has application in wide areas including digital subscriber lines, broadband communication applications including 4G, cellular networks based on longterm evolution (LTE), high-definition television broadcasting and optical communication [1].OFDM is suitable technique for high speed data transmission due to its inherent advantages of reduced intersymbol interference and high spectral efficiency [2].The optical fibers offer very high bandwidth but with the swift increase in internet data, it is requisite to use fiber bandwidth efficiently. OFDM is considered as an emerging technique for high speed optical communication [1].

\section{OFDM}

OFDM is modulation technique in which the bit stream to be transmitted is split into several parallel low data rate bit streams. The available frequency spectrum is divided into sub-channels and each low rate bit stream is transmitted over one sub-channel. OFDM offers a spectral efficiency advantage as it eliminates the spectral guard bands between the subcarriers by using the principle of orthogonality [1]. To obtain orthogonality, subcarrier frequencies, $f_{n}(n=0,1,2$ ...N-1) have to chosen so that the condition given in (1) is satisfied.

$$
\int_{0}^{\mathrm{T}} \mathrm{s}_{\mathrm{n}}(\mathrm{t}) \mathrm{s}_{\mathrm{n}^{\prime}}(\mathrm{t}) d \mathrm{t}=0
$$

This condition can be satisfied if the subcarrier frequencies are chosen to be integral multiples of $1 / \mathrm{T}$ as given in (2). The term $f_{R}$ represents the radio frequency which is used for frequency up conversion.

$$
\begin{gathered}
f_{n}=\frac{n}{T}+f_{R} \\
\text { for } n=0,1,2, \ldots N-1
\end{gathered}
$$

Mathematically, the OFDM signal can be expressed as

$$
X(k)=\left(\frac{1}{\sqrt{N}}\right) \sum_{k=0}^{N-1} x(n) e^{\frac{j 2 \pi n k}{N}}
$$

$$
\text { for } 0 \leq \mathrm{k} \leq \mathrm{N}-1
$$

Where, $\mathrm{X}(\mathrm{k})$ is transmitted information symbol for the kth subcarrier in the OFDM symbol which can be realized using Inverse Fast Fourier Transform(IFFT). At the receiver, Fast Fourier Transform (FFT) is used to recover the data.

The block diagram of an optical OFDM system is shown in Fig 1.

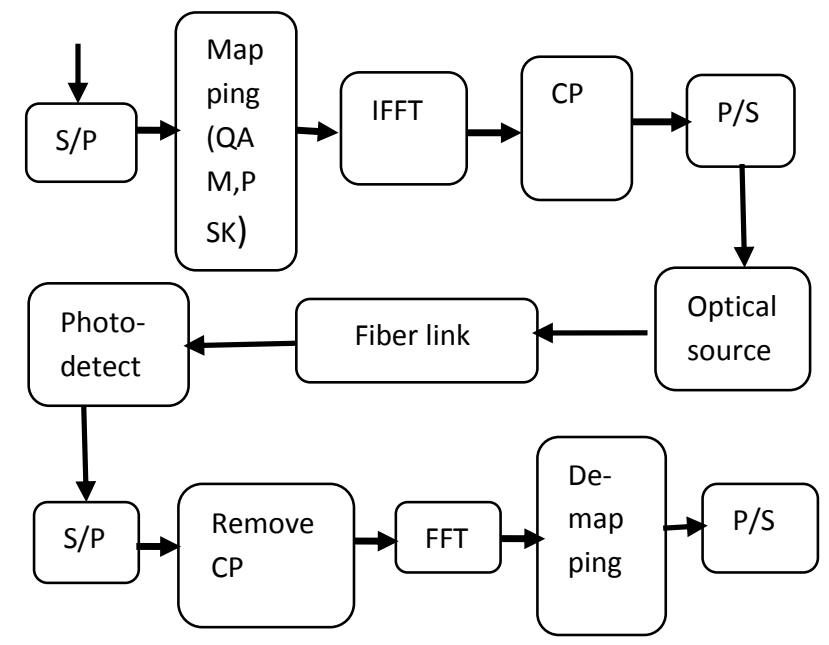

Fig 1 Block diagram of optical OFDM system

At the transmitter, high rate digital data stream is first divided into $\mathrm{N}$ parallel streams. Each stream is mapped to symbols using some modulation scheme which are then modulated onto the orthogonal subcarriers using IFFT which generates OFDM signal. A cyclic prefix is then added to the OFDM symbol by adding samples from end of the sequence to the start of the sequence. This is done to reduce intersymbol interference [1].The data sequence is then converted to analog domain using digital to analog converter (DAC). The OFDM sequence is then used to modulate the properties of light emitted by optical source. The output of optical source is then transmitted over optical fiber link.

At receiver, the optical signal is detected using photodiodes. The detection can be classified into two types. The two 
methods are Direct Detection (DD) using intensity modulation and Coherent Detection (CO).Direct detection receiver uses measurement of signal energy for reception of signals and coherent detection method requires a local oscillator to generate the transmitted frequency [3]. After detection, received signal is down-converted to baseband frequency and converted form serial to parallel form . Then it is converted from analog to digital using an analog-to- digital converter (ADC). The cyclic prefix is removed thereafter and samples are fed into the Fast Fourier transform (FFT) to be converted to frequency domain. Finally, data is detected by demapping and parallel to serial conversion.

\section{PAPR}

The high Peak to Average Power Ratio (PAPR) is one of the major drawbacks of the OFDM modulation [2]. Since an OFDM signal is formed by adding a number of data modulated independent subcarriers, it can produce a large PAPR when all subcarriers add up coherently. The expression for PAPR of an OFDM signal can be written as below.

$$
\operatorname{PAPR}=\frac{\max \left([\mathrm{x}(\mathrm{t}) \mid)^{2}\right.}{\mathrm{E}\left(|\mathrm{x}(\mathrm{n})|^{2}\right)}
$$

The major drawback of high PAPR is that it enhances the non linear effects of optical fiber. The effective refractive index of optical fibre can be expressed in terms of the effective core area $\left(\mathrm{A}_{e f f}\right)$ and input power $(P)$ as,

$$
\mathrm{n}_{\text {eff }}=\mathrm{n}_{1}+\mathrm{n}_{1} \mathrm{P} / \mathrm{A}_{\text {eff }}
$$

From (7), it is clear that, the non linearity in the fiber place a constraint on the maximum optical power that can be launched into the fibre. Thus, by reducing the value of the PAPR of an OFDM signal, the power level of the optical signal after modulation with the LASER source remains fairly low. High PAPR also requires a high dynamic range of linear power amplifier, Digital to Analog/ Analog to Digital converters for operation in linear region.

For an OFDM signal with $\mathrm{N}$ subcarriers, the maximum value of PAPR can be calculated using (6).

$$
\mathrm{PAPR}_{\max }=10 \log 10 \mathrm{~N}(d B)
$$

From (6), it can be seen that PAPR increases with increase in number of subcarriers. In next section, major PAPR reduction techniques have been discussed.

\section{PAPR REDUCTION TECHNIQUES}

In order to reduce the high PAPR value in optical OFDM systems, various techniques have been proposed in literature. Most important of these are signal clipping, companding, SLM, PTS, non linear sliding norm transforms etc. These techniques are discussed below.

\subsection{Signal Clipping}

Amplitude clipping is a simple technique to reduce the PAPR of OFDM signal. A predefined value of the amplitude is used to limit the peak value of the input signal. Signal having values higher than this threshold value are clipped to the threshold value.In the optical OFDM system, the signal is clipped at zero level first and the positive and real samples $x$ are clipped at an amplitude A as follows.

$$
f(x)=\{\text { A when } x>A,
$$

$$
\mathrm{x} \text { when } 0 \leq \mathrm{x}<\mathrm{A}\}
$$

The main problem in this case is that the amplitude clipping introduces undesired clipping noise giving rise to in-band and out of band distortion [4]. Out of band radiation distortion can be reduced by filtering after clipping but this may result in peak regrowth. Iterative clipping and filtering can be used to overcome this problem [4], [5].

\subsection{Companding based PAPR reduction}

The companding technique is a pre-distortion process in time domain. Using his technique, the amplitude of the small signal is enlarged while the large signal remains almost the same [6]. The companding is performed using $\mu$-law given by (8).

$$
y=P R \times x_{\text {peak }} \times \frac{\log \left(1+\mu \frac{|\mathrm{x}|}{\mathrm{PR} \times \mathrm{x}_{\text {peak }}}\right)}{\log (1+\mu)} \operatorname{sgn}(\mathrm{x})
$$

Here, $P R$ (peak ratio) is the ratio of peak amplitude of compressor to the peak of actual signal and $\mathrm{x}$ is the instantaneous amplitude of input signal. At, receiver, expanding is achieved by the inverse of equation.

The gain of PAPR reduction and noise enhancement are increased as $\mu$ is increased. Choosing PR $>1$ results in a gain greater than one for the peaks and a very high gain for the lower amplitude signals. Hence, signal amplitude is redistributed after transformation which results in reduced PAPR. Also, there is increase in noise due to companding for a constant value of signal-to-noise ratio. Hence it is important to choose values of companding parameters to avoid significant noise [6]

\subsection{Selected mapping (SLM)}

In selected mapping method, firstly $M$ statistically independent sequences which represent the same information are generated. The resulting $M$ independent data blocks $\boldsymbol{S}_{\mathrm{m}}=\left[S_{m, 0}, S_{m, 1}, \ldots, S_{m, N-1}\right]^{\mathrm{T}}, \quad m=1,2, \ldots, M$ are obtained by multiplying the original sequence with $M$ uncorrelated sequence $\boldsymbol{P}_{\mathrm{m}}$. These are then forwarded into IFFT operation simultaneously. And then the PAPR of these M vectors are calculated separately. The sequence with the smallest PAPR is then selected for final serial transmission.

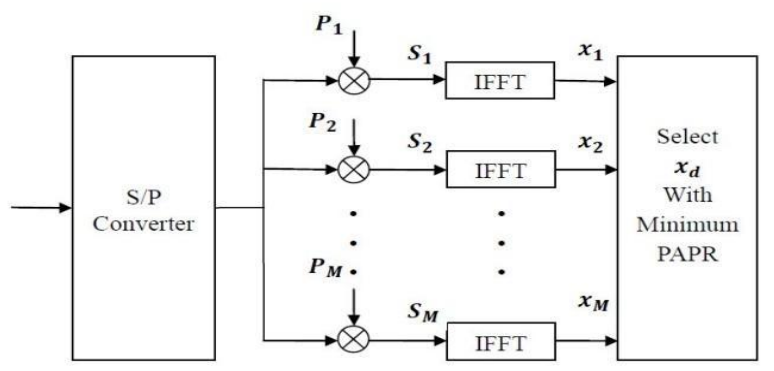

Fig 2 Block diagram of SLM technique for PAPR reduction

At the receiver, in order to correctly demodulate the received signal, it becomes indispensable to know which sequence is linked to the smallest PAPR among M different. Hence, the receiver needs the information about selected phase vector sequence and ensure that the vector sequence is received correctly [7]. This can degrade the spectral efficiency of the system 


\subsection{Partial transmit sequence (PTS)}

The basic scheme behind partial transmit sequences algorithm is to divide the original OFDM sequence into several subsequences. Each sub-sequence is multiplied by different weights and then an optimum value is chosen.

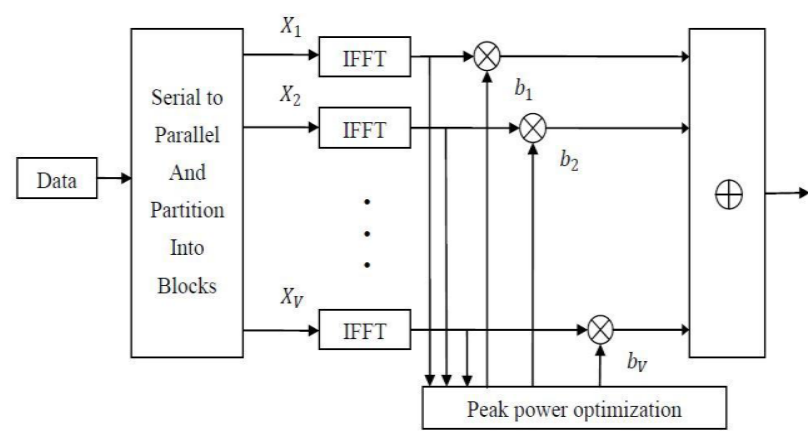

Fig.3. Block diagram of PTS technique for PAPR reduction

Data information in frequency domain $\mathbf{X}$ is separated into $\mathrm{V}$ non-overlapping sub-blocks and each sub-block vectors has the same size $N$. Consequently, for every sub-block, it has N/V nonzero elements and set the rest part to zero. The subblock vector is given by

$$
\widehat{\mathrm{X}}=\sum_{v=1}^{\mathrm{v}} \mathrm{b}_{v} \mathrm{x}_{v}
$$

where $b_{v}=e\left(j \varphi_{v}\right), \varphi_{v} \in(0,2 \pi v=1,2, \ldots, V)$ is a weighting factor used for phase rotation. The signal in time domain is obtained by applying IFFT operation on $X_{v}$. One suitable factor combination $\mathbf{b}=\left[b_{1}, b_{2}, \ldots, b_{v}\right]$ has to be selected which makes the result achieve optimum [8] which is obtained using (10) as given below.

$\mathrm{b}=\left[\mathrm{b}_{1}, \mathrm{~b}_{2}, \ldots \mathrm{b}_{v}\right]$
$=\operatorname{argmin}_{\left(\mathrm{b}_{1}, \mathrm{~b}_{2}, \ldots \ldots ., \mathrm{b}_{v}\right)}\left(\max _{1 \leq \mathrm{n} \leq \mathrm{N}}\left|\sum_{v=1}^{\mathrm{V}} \mathrm{b}_{v} \mathrm{X}_{v}\right|^{2}\right)$

Here $\operatorname{argmin}(\cdot)$ is the judgment condition that gives the minimum value of function. In this way best $b$ can be found so as to optimize the PAPR performance. The additional cost have to pay is the extra V-1 times IFFTs operation [8].

\subsection{Sliding Norm Transform (SNT)}

Another method to reduce PAPR is SNT. The L2-by-3 transform is a particular kind of discrete sliding norm transform. It uses three samples, $\mathrm{x}_{\mathrm{n}-1}, \mathrm{x}_{\mathrm{n}}, \mathrm{x}_{\mathrm{n}+1}$, and a controlling parameter, $\alpha$, in each sliding window to calculate the output samples [10].

$$
\mathrm{y}_{\mathrm{n}}=\frac{\mathrm{x}_{\mathrm{n}}}{\sqrt{\alpha+\mathrm{x}_{\mathrm{n}-1}^{2}+\mathrm{x}_{\mathrm{n}}^{2}+\mathrm{x}_{\mathrm{n}+1}^{2}}}
$$

At the receiver, after optical to electrical conversion, Inverse Sliding norm transform is applied as given below.

$$
\mathrm{x}_{n}=\left(\sqrt[3]{\mathrm{x}_{\mathrm{n}}^{3}}\right) \cdot \operatorname{sign}\left(y_{n}\right)
$$

Then, the FFT is applied to each signal and original signal is recovered after the demapping process.

The performance of the system is improved in terms out-ofband distortion and there is no loss in data rates because the proposed method does not require any side information [10]

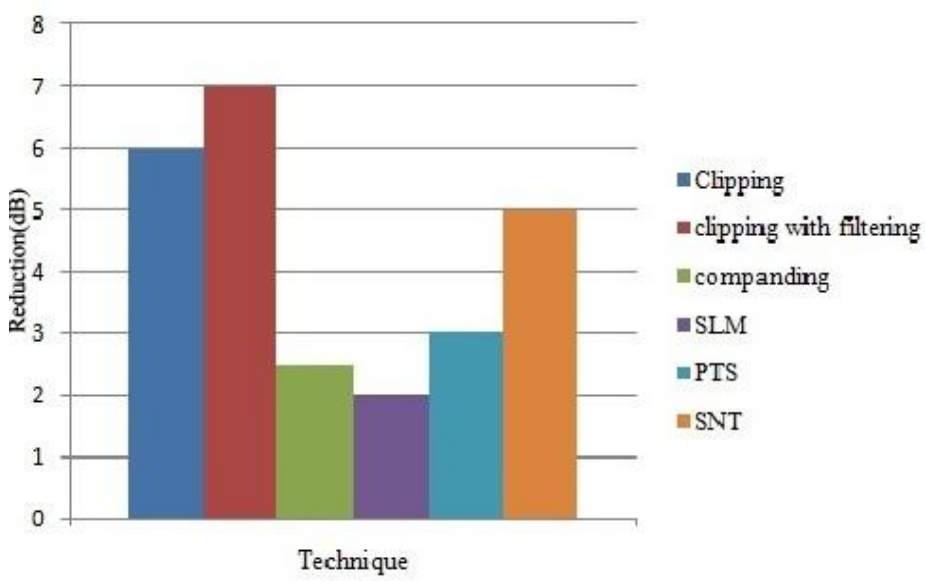

Fig 4 Reduction in PAPR using various techniques in dB

\section{CONCLUSION}

OFDM is a promising technique for very high speed optical communication. OFDM offers spectral efficiency as it eliminates the need of guard bands between the subcarriers. Its major advantage is reduction in intersymbol interference. Optical OFDM systems with coherent detection method improve receiver sensitivity over systems with direct detection. However, OFDM has inherent drawback of high Peak to Average Power Ratio (PAPR) which arises due to a large number of subcarriers which when added coherently produce a large peak power. High PAPR means a large dynamic range of devices like amplifiers is required to avoid nonlinear effects which degrade the optical signal to noise ratio. Various methods have been proposed for reduction of PAPR in optical communication which include predistortion, coding and scrambling. This paper carries a view of various PAPR reduction techniques. Figure 4 shows the reduction in PAPR using different techniques. Clipping and companding exhibit good PAPR reduction Capabilities but introduce unwanted distortion.SLM and PTS require extra information for recovery of data. Sliding Norm based transform does not require side information and give good values of PAPR reduction for some values of parameter $\alpha$. Table 1 summarizes the merits and demerits of various PAPR reduction techniques. These techniques can be used in optical OFDM systems for performance improvement of the system.

Table 1. Summary of PAPR reduction technique

\begin{tabular}{|l|l|l|}
\hline S No. & Technique & \multicolumn{1}{c|}{ Merits and demerits } \\
\hline 1. & $\begin{array}{l}\text { Clipping/ } \\
\text { predistortion }\end{array}$ & Simple technique. Reduces PAPR significantly but introduces clipping noise. \\
\hline
\end{tabular}




\begin{tabular}{|l|l|l|}
\hline 2. & Companding & Does not predistort data. Introduces spectrum side lobes hence BER degradation. \\
\hline 3. & Selective mapping & $\begin{array}{l}\text { Effective technique. But side information is needed for recovery of data, hence loss in data } \\
\text { rates. }\end{array}$ \\
\hline 4. & $\begin{array}{l}\text { Partial transmit } \\
\text { sequence }\end{array}$ & $\begin{array}{l}\text { Value of parameter b can be found out for optimized PAPR. But IFFT has to be performed V-1 } \\
\text { times. }\end{array}$ \\
\hline 5. & $\begin{array}{l}\text { Sliding norm } \\
\text { transforms }\end{array}$ & $\begin{array}{l}\text { Doesn't require side information. But gives good PAPR reduction for some values of } \\
\text { parameter } \alpha .\end{array}$ \\
\hline
\end{tabular}

\section{REFERENCES}

[1] Neda Cvijetic,"OFDM for Next-Generation Optical Access Networks." Journal of lightwave technology, vol. 30, no. 4, February 15, 2012.

[2] Armstrong, J., "OFDM for Optical Communications." Journal of Lightwave Technology, 27(3), pp.189-204, 2009.

[3] Kahn, Joseph,"Modulation and detection techniques for optical communication systems." Coherent Optical Technologies and Applications. Optical Society of America,2006.

[4] Lin CHEN, Yong FANG," PAPR Reduction in Optical OFDM Systems Using Asymmetrically Clipping and Filtering Technique."Journal of Computational Information Systems 8: 7 2733-2740,2012.

[5] Yu, Zhenhua, Robert J. Baxley, and G. Tong Zhou. "Iterative Clipping for PAPR Reduction in Visible Light OFDM Communications." Military Communications Conference (MILCOM), 2014 IEEE. IEEE, 2014.

[6] Hwan Seok Chung, Sun Hyok Chang, and Kwangjoon Kim,"Companding Transform based SPM Compenstaion in Coherent Optical OFDM transmission." Optical Society of America,2011.

[7] Yufeng Shao, Nan Chi, Jingyuan Fan, and Wuliang Fang,"Generation of 16-QAM-OFDM Signals using Selected Mapping Method and Its Application in Optical Millimeter-Wave Access System." IEEE Photonics Technology Letters, VOL. 24, NO. 15, August 1, 2012.

[8] Wang, Jianping, Ying Guo, and Xianwei Zhou, "PTSclipping method to reduce the PAPR in ROF-OFDM system." Consumer Electronics, IEEE Transactions on 55.2 (2009): 356-359, 2009.

[9] Dursun, S. \& Grigoryan, A.M.," Nonlinear L2-by-3 transform for PAPR reduction in OFDM systems."Computers \& Electrical Engineering, 36(6), pp.1055-1065, 2010.

[10] M.Hariharan, M. Gurunathan, A. Gopi, M. Palanivelan \& Sheila Anand," Non Linear Transform Technique to Optimize PAPR in Optical OFDM (O-OFDM) Systems." International Conference on Computer Science and Information Technology, 10, March 2013. 